INPLASY

PROTOCOL

To cite: Chen et al. The association between LSP1 rs3817198(T>C) polymorphism and breast cancer: a metaanalysis. Inplasy protocol 2021120127. doi:

10.37766/inplasy2021.12.0127

Received: 28 December 2021

Published: 28 December 2021

Corresponding author:

Yingliang Li

drliyingliang1977@163.com

Author Affiliation:

General Surgery Department, First Affiliated Hospital of Nanchang University.

Support: Project grant fund.

Review Stage at time of this submission: Preliminary searches.

Conflicts of interest: None declared.

\section{The association between LSP1 rs3817198(T $>C$ ) polymorphism and breast cancer: a meta-analysis}

Chen, J1; Xie, Y2; Liu, R3; Liu, Z4; Long, X5; Huang, J6; Xiong, H7; $\mathrm{Li}, \mathrm{Y} 8$;

Review question / Objective: The correlation of single nucleotide polymorphisms (SNPs) in leukocyte-specific protein 1 (LSP1) with breast cancer susceptibility has been reported in many studies, especially $r s 3817198(T>C)$. Nevertheless, findings have been inconsistent and even contradictory. Therefore, we will performed meta-analysis to comprehensively evaluate the extent of association of rs3817198 polymorphism with breast cancer.

Condition being studied: Breast cancer is the top cause of death in women [1], and the LSP1 gene has been reported to be a susceptibility gene for breast cancer [2]. Several studies have reported the association of LSP1 rs3817198 with breast cancer, including genome-wide association analysis studies (GWAS), but the conclusions are different. We are planning to take a meta-analysis to comprehensively assess the association of rs3817198 with breast cancer.

INPLASY registration number: This protocol was registered with the International Platform of Registered Systematic Review and Meta-Analysis Protocols (INPLASY) on 28 December 2021 and was last updated on 28 December 2021 (registration number INPLASY2021120127).

\section{INTRODUCTION}

Review question / Objective: The correlation of single nucleotide polymorphisms (SNPs) in leukocytespecific protein 1 (LSP1) with breast cancer susceptibility has been reported in many studies, especially rs3817198(T>C).
Nevertheless, findings have been inconsistent and even contradictory. Therefore, we will performed meta-analysis to comprehensively evaluate the extent of association of rs3817198 polymorphism with breast cancer. 
Condition being studied: Breast cancer is the top cause of death in women [1], and the LSP1 gene has been reported to be a susceptibility gene for breast cancer [2]. Several studies have reported the association of LSP1 rs3817198 with breast cancer, including genome-wide association analysis studies (GWAS), but the conclusions are different. We are planning to take a meta-analysis to comprehensively assess the association of rs3817198 with breast cancer.

\section{METHODS}

Search strategy: We are going to run a comprehensive literature search to include eligible studies based on four databases (Pubmed, Embase, Web of Science, and NCKI) up to October 2020 without language restrictions. The search terms of Pubmed are as follows: ("Breast Neoplasms"[MeSH] OR "breast neoplasm"[Title/Abstract] OR "breast tumor"[Title/Abstract] OR "breast cancer"[Title/Abstract] OR "mammary cancer"[Title/Abstract] OR "breast malignant neoplasm"[Title/Abstract] OR "breast malignant tumor"[Title/Abstract] OR "human mammary carcinoma"[Title/ Abstract] OR "mammary neoplasm"[Title/ Abstract]) OR "breast carcinoma"[Title/ Abstract]) AND ("lymphocyte specific protein 1"[Title/Abstract] OR "Isp1 protein"[Title/Abstract] OR "LSP1"[Title/ Abstract] OR "rs3817198"[Title/Abstract]) AND ("polymorphism, single nucleotide"[MeSH] OR "Mutation"[MeSH] OR "Genetic Variation"[MeSH] OR "Alleles"[MeSH] OR "SNPs"[Title/Abstract] OR "Polymorphism"[Title/Abstract]) AND ("Case-Control Studies"[MeSH] OR "Cohort Studies"[MeSH]). And the search strategy for the other three databases are similar.

Participant or population: Inclusion criteria: Patients diagnosed with breast cancer. Exclusion criteria: Breast cancer patients who have received radiotherapy.

Intervention: The associations between LSP1 rs3817198 polymorphism and cancer susceptibility.
Comparator: Inclusion criteria: People with no history of breast cancer Exclusion criteria: Patients with other malignant tumors.

Study designs to be included: Case-control studies.

Eligibility criteria: The inclusion criteria for this meta-analysis are as follows: (a) studies regarding the Lsp1 rs3817198(T>C) polymorphism and breast cancer; (b) studies designed as case-control studies, cohort studies, or nested case-control studies; (c) studies with a case-group of patients with pathologically diagnosed breast cancer. Exclusion criteria are: (a) duplicate literature; (b) lack of data; (c) non-human studies.

Information sources: 1 .We are going to run a comprehensive literature search to include eligible studies based on four databases (Pubmed, Embase, Web of Science, and NCKI). 2.Hand search based on the reference of relevant article. 3. Grey literature.

Main outcome(s): Association of LSP1 rs3817198(T>C) with breast cancer susceptibility.

Additional outcome(s): If feasible, we will perform a subgroup analysis to analyze the effect of ethnicity, gender and other factors.

Quality assessment / Risk of bias analysis: Risk of bias assessment: We will evaluate publication bias with funnel plots and Egger's test. Quality assessment: Two researchers will evaluate the quality of the included studies according to the Newcastle-Ottawa Scale (NOS).

Strategy of data synthesis: We will assess the assciation of LSP1 rs3817198 polymorphism and breast cancer by OR and $95 \% \mathrm{Cl}$ in five models (dominant, recessive, heterozygous, homozygous, and additive models). And the Cochran's $Q$ test and the $I^{2}$ statistic will be used to evaluate the level of heterogeneity of the included original studies; if the $P$-value of $Q$ test is 


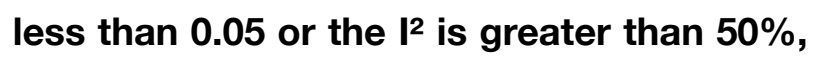
significant heterogeneity is considered to exist and a random-effects model is used to combine the data; otherwise, a fixedeffects model is used. Meta-regression and subgroup analysis will be performed to explore sources of heterogeneity and publication bias will be assessed with funnel plots and Egger's test. Sensitivity analysis will be used to assess the stability of the findings. All data analyses above were performed on STATA 16.0.

Subgroup analysis: Subgroup analyses will be conducted according to the results of the meta-regression. If feasible, we will perform subgroup analyses of the included studies for ethnicity, control source and so no to further probe sources of heterogeneity.

Sensitivity analysis: The leave-one-out method will be implemented to perform sensitivity analyses by sequentially eliminating each study and recalculating ORs and $95 \%$ Cls to assess the stability of the results.

Language: No.

Country(ies) involved: China.

Other relevant information: References - [1] Torre LA, Bray F, Siegel, et al. Global cancer statistics 2012 [J]. CA Cancer J Clin, 2015, 65 (2): 87-108. [2] Easton DF, Pooley KA, Dunning $A M$, et al. Genome-wide association study identifies novel breast cancer susceptibility loci [J]. Nature, 2007, 447 (7148): 10U7-1087.

Keywords: single nucleotide polymorphisms, SNP, leukocyte-specific protein 1, LSP1, rs3817198, meta-analysis, breast cancer.

Contributions of each author:

Author 1 - Jian Chen.

Email: 3150246768@qq.com

Author 2 - Yufen Xie.

Email; 942239009@qq.com

Author 3 - Ruihao Liu.

Email: drliuruihao@163.com

Author 4 - Zhigao Liu.
Email: Izg6858@163.com

Author 5 - Xiaozhou Long.

Email: 352940983@qq.com

Author 6 - Jinlong Huang.

Email: drhuangjinlong@163.com

Author 7 - Haiwei Xiong.

Email: drxionghaiweisx@163.com

Author 8 - Yinliang Li - Corresponding Author.

Email: drliyingliang1977@163.com 